\title{
Exact Ground States of Frustrated Spin-1 Ising-Heisenberg and Heisenberg Ladders in a Magnetic Field
}

\author{
J. Strě̌Ka ${ }^{a, *}$, F. MiChAud ${ }^{b}$, F. Mila ${ }^{b}$ \\ ${ }^{a}$ Institute of Physics, Faculty of Science, P.J. Šafárik University, Park Angelinum 9, 04001 Košice, Slovakia \\ ${ }^{b}$ Institute of Theoretical Physics, Ecole Polytechnique Fédérale de Lausanne, CH-1015 Lausanne, Switzerland \\ Ground states of the frustrated spin-1 Ising-Heisenberg two-leg ladder with Heisenberg intra-rung coupling \\ and only Ising interaction along legs and diagonals are rigorously found by taking advantage of local conservation of \\ the total spin on each rung. The constructed ground-state phase diagram of the frustrated spin-1 Ising-Heisenberg \\ ladder is then compared with the analogous phase diagram of the fully quantum spin-1 Heisenberg two-leg ladder \\ obtained by density matrix renormalization group (DMRG) calculations. Both investigated spin models exhibit \\ quite similar magnetization scenarios, which involve intermediate plateaux at one-quarter, one-half and three- \\ quarters of the saturation magnetization.
}

DOI: $10.12693 /$ APhysPolA.126.24

PACS: 05.50.+q, 75.10.Jm , 75.10.Kt , 75.60.Ej

\section{Introduction}

Over the last few decades, quantum spin ladders have been actively studied mainly in connection with spinliquid behaviour, quantum critical points and superconductivity under hole doping of some cuprates (see Ref. [1] for a review). In particular, the frustrated spin-1/2 Heisenberg two-leg ladder exhibits a striking dimerized ground state [2] and a low-temperature magnetization process with an intermediate plateau and magnetization jumps [3].

Another challenging topic of current research interest consists of the theoretical investigation of related models such as the quantum spin-1 Heisenberg two-leg ladder $[4,5]$. The main goal of the present work is to find the exact ground states of a simpler spin-1 Ising-Heisenberg ladder and to contrast them with the respective ground states of the pure quantum spin-1 Heisenberg ladder. Note that the former model is analytically tractable using the procedure developed in Refs. [6, 7] and it brings insight into the relevant behaviour of the latter not fully integrable model.

\section{Frustrated Ising-Heisenberg ladder}

Consider first the frustrated spin-1 Ising-Heisenberg ladder with the Heisenberg intra-rung interaction and the unique Ising interaction along the legs and diagonals. The total Hamiltonian of the investigated model is given by

$$
\begin{aligned}
\hat{H} & =\sum_{i=1}^{N}\left[J \hat{\boldsymbol{S}}_{1, i} \cdot \hat{\boldsymbol{S}}_{2, i}+J_{1}\left(\hat{S}_{1, i}^{z}-h\left(\hat{S}_{1, i}^{z}+\hat{S}_{2, i}^{z}\right)\right.\right. \\
& \left.\left.+\hat{S}_{2, i}^{z}\right) \cdot\left(\hat{S}_{1, i+1}^{z}+\hat{S}_{2, i+1}^{z}\right)\right],
\end{aligned}
$$

where $\hat{\boldsymbol{S}}_{\alpha, i} \equiv\left(\hat{S}_{\alpha, i}^{x}, \hat{S}_{\alpha, i}^{y}, \hat{S}_{\alpha, i}^{z}\right)$ denotes spatial components of the spin-1 operator, the former suffix $\alpha=1$ or

\footnotetext{
*corresponding author; e-mail: jozef.strecka@upjs.sk
}

2 enumerates the leg and the latter suffix specifies a lattice position within a given leg. The coupling constant $J$ denotes the isotropic Heisenberg intra-rung interaction, the parameter $J_{1}$ determines the Ising interaction along the legs and diagonals, $h$ is an external magnetic field.

For further convenience, let us introduce the spin operator $\hat{\boldsymbol{T}}_{i}=\hat{\boldsymbol{S}}_{1, i}+\hat{\boldsymbol{S}}_{2, i}$, which corresponds to the total spin angular momentum of the $i$ th rung. It can be easily proved that the operators $\hat{\boldsymbol{T}}_{i}^{2}$ and $\hat{T}_{i}^{z}$ commute with the Hamiltonian (1), i.e. $\left[\hat{\boldsymbol{T}}_{i}^{2}, \hat{H}\right]=\left[\hat{T}_{i}^{z}, \hat{H}\right]=0$, which means that the total spin of a rung and its $z$ component represent conserved quantities with well defined quantum numbers. The complete energy spectrum of the frustrated spin-1 Ising-Heisenberg ladder then readily follows from the relation

$$
\begin{gathered}
E=-2 N J+\frac{J}{2} \sum_{i=1}^{N} T_{i}\left(T_{i}+1\right)+ \\
J_{1} \sum_{i=1}^{N} T_{i}^{z} T_{i+1}^{z}-h \sum_{i=1}^{N} T_{i}^{z},
\end{gathered}
$$

which depends just on the quantum numbers $T_{i}=0,1,2$ and $T_{i}^{z}=-T_{i},-T_{i}+1, \ldots, T_{i}$ determining the eigenvalues of the total spin of the $i$ th rung and its $z$ spatial projection, respectively. Using this procedure, the spin-1 IsingHeisenberg two-leg ladder has been rigorously mapped to some classical chain of composite spins and accordingly, we can readily find all available ground states by looking for the lowest-energy state of Eq. (2).

\section{Frustrated Heisenberg ladder}

Next, we will also consider the frustrated spin-1 Heisenberg two-leg ladder defined by the Hamiltonian

$$
\begin{aligned}
\hat{H} & =\sum_{i=1}^{N}\left[J \hat{\boldsymbol{S}}_{1, i} \cdot \hat{\boldsymbol{S}}_{2, i}-h\left(\hat{S}_{1, i}^{z}+\hat{S}_{2, i}^{z}\right)\right. \\
& \left.+J_{1}\left(\hat{\boldsymbol{S}}_{1, i}+\hat{\boldsymbol{S}}_{2, i}\right) \cdot\left(\hat{\boldsymbol{S}}_{1, i+1}+\hat{\boldsymbol{S}}_{2, i+1}\right)\right],
\end{aligned}
$$

which represents the pure quantum analogue of the frustrated spin-1 Ising-Heisenberg ladder discussed previously. Taking advantage of the definition for the total 
spin of each rung, the Hamiltonian (3) of frustrated spin1 Heisenberg two-leg ladder can be rewritten into the form

$$
\begin{aligned}
& \hat{H}=-2 N J+\frac{J}{2} \sum_{i=1}^{N} \hat{\boldsymbol{T}}_{i}^{2}+ \\
& J_{1} \sum_{i=1}^{N} \hat{\boldsymbol{T}}_{i} \cdot \hat{\boldsymbol{T}}_{i+1}-h \sum_{i=1}^{N} \hat{T}_{i}^{z} .
\end{aligned}
$$

According to Eq. (4), the frustrated spin-1 Heisenberg ladder can be rigorously decomposed into the direct sum of quantum spin chains with spin 0,1 or 2 at each site. The ground state of such a system can be shown to be either a homogeneous chain, with the same spin at all sites, or a chain with alternating spins on every other site. Comparing the energy of the different chains, obtained either analytically or using DMRG simulations, the exact ground-state phase diagram of the frustrated

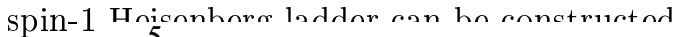
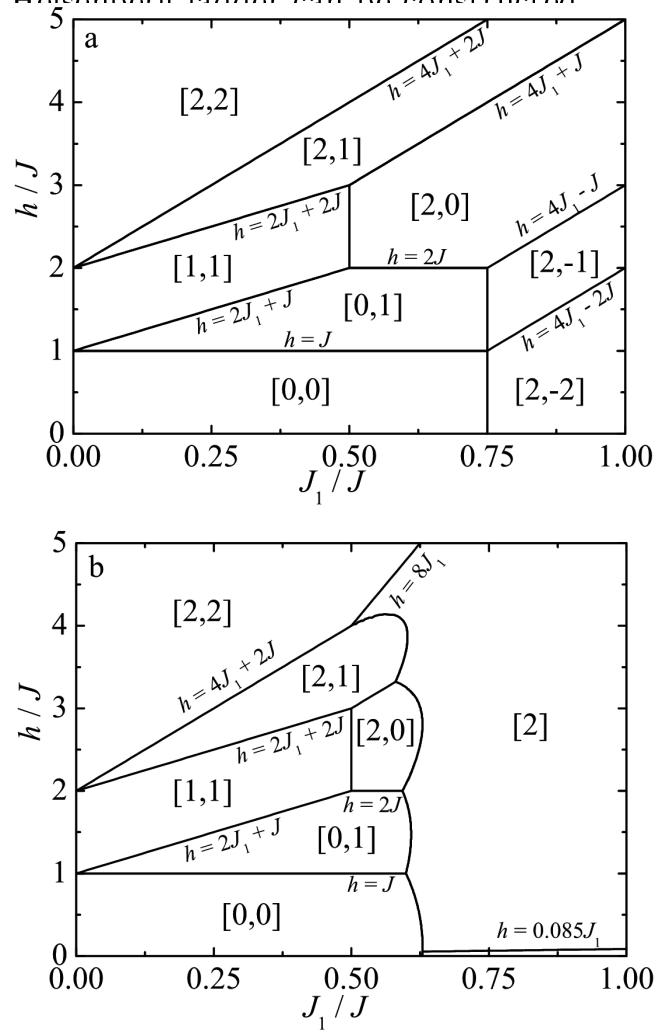

Fig. 1. Ground-state phase diagrams of the frustrated spin-1 ladder described within: (a) the Ising-Heisenberg model; (b) the pure Heisenberg model. For details see the text.

\section{Results and discussion}

The constructed ground-state phase diagrams of the frustrated spin-1 Ising-Heisenberg and Heisenberg ladders are depicted in Fig. 1a and Fig. 1b, respectively. The ground states of the spin-1 Ising-Heisenberg ladder can be discerned according to the $z$ projection of the total spin on two consecutive rungs $\left[T_{i}^{z}, T_{i+1}^{z}\right]$, because $T_{i}=\left|T_{i}^{z}\right|$ holds for all available ground states. The quan- tum ground states $[0,0],[0,1],[1,1],[2, \pm 1]$ and $[2,0]$ represent six different phases, whereas $T_{i}=T_{i}^{z}=0$ implies a formation of two singlets on the $i$ th rung, $T_{i}=\left|T_{i}^{z}\right|=1$ entails only one singlet and $T_{i}=\left|T_{i}^{z}\right|=2$ denotes fully polarized rungs without singlets. Besides, the two ground states $[2,2]$ and $[2,-2]$ are pertinent to the classical ferromagnetic and antiferromagnetic ordering of fully polarized rungs. The magnetization normalized with respect to its saturation equals zero for $[0,0]$ and $[2,-2]$, one-quarter for $[0,1]$ and $[2,-1]$, one-half for $[1,1]$ and $[2,0]$, three-quarters for $[2,1]$ and unity for $[2,2]$. Altogether, it can be concluded that the frustrated spin-1 Ising-Heisenberg ladder always exhibits a stepwise magnetization curve, which involves intermediate plateaux at one-quarter, one-half and three-quarters of the saturation magnetization that are however of different origin.

It is quite clear from Fig. 1b that the ground-state phase diagram of the pure quantum Heisenberg ladder exactly coincides with that of the Ising-Heisenberg ladder just for sufficiently weak inter-rung interactions $J_{1} / J \leq 0.5$. A relatively good agreement between both phase diagrams is still observed in the parameter space $0.5 \leq J_{1} / J \leq 0.63$, where the gapless phase [2] with a continuously varying magnetization is present between the intermediate plateaux instead of direct magnetization jumps. The gapless phase [2] corresponds to the Luttinger-liquid phase of the effective spin-2 quantum Heisenberg chain. Finally, the gapped Haldane phase of the effective spin-2 quantum Heisenberg chain emerges for $J_{1} / J \geq 0.63$ at sufficiently low fields.

In conclusion, we have rigorously found the ground states of the frustrated spin-1 Ising-Heisenberg and Heisenberg ladders in a magnetic field. It has been verified that the Ising-Heisenberg ladder always exhibits a stepwise magnetization curve with three different intermediate plateaus, while the same quantum ground states can be identified in the pure quantum Heisenberg ladder provided the intra-rung coupling is sufficiently large.

\section{Acknowledgments}

This work was financially supported by the projects VEGA 1/0234/12, APVV-0132-11, and ERDF EU grant ITMS 26220120047 (activity 3.2). F. Michaud thanks S.R. Manmana for providing his DMRG code.

\section{References}

[1] M.T. Batchelor, X.W. Guan, N. Oelkers, Z. Tsuboi, Adv. Phys. 56, 465 (2007).

[2] M.P. Gelfand, Phys. Rev. B 43, 8644 (1991).

[3] A. Honecker, F. Mila, M. Troyer, Eur. Phys. J. B 15, 227 (2000).

[4] V. Ravi Chandra, N. Surendan, Phys. Rev. B 74, 024421 (2006).

[5] F. Michaud, T. Coletta, S.R. Manmana, J.D. Picon, F. Mila, Phys. Rev. B 81, 014407 (2010).

[6] J.H. Barry, M. Meisel, Phys. Rev. B 58, 3129 (1998).

[7] O. Rojas, J. Strečka, M. Lyra, Phys. Lett. A 377, 920 (2013). 